\title{
Fabrication of Microenvironments with Different Geometrical Features for Cell Growth Studies
}

\author{
O. I. Avila ${ }^{*}$, A. J. G. Otuka ${ }^{*}$, V. Tribuzi ${ }^{*}$ L. M. Freitas ${ }^{* *}$, R. B. Serafim ${ }^{* *}$, M. H. Moraes ${ }^{* * *}$, E. M. Espreafico ${ }^{* * *}$, V. \\ Valente $^{* * * * * * * *}$, C. R. Fontana ${ }^{* * *}$, C. R. Mendonça* \\ *Instituto de Física de São Carlos, Universidade de São Paulo, 13560-970 São Carlos, SP, Brazil \\ E-mail: crmendon@ifsc.usp.br \\ **Faculdade de Ciências Farmacêuticas, Univ Estadual Paulista (UNESP), Rua Expedicionarios do Brasil \\ 1621, \\ Araraquara, Sao Paulo 14801-960, Brazil \\ ${ }^{* * * *}$ Faculdade de Medicina de Ribeirão Preto, Universidade de São Paulo (USP), \\ Avenida dos Bandeirantes, 3900, CEP 14049-900, Ribeirão Preto, São Paulo, Brasil \\ ${ }^{* * * *}$ CISBi (Center for Integrative Systems Biology), NAP/USP
}

\begin{abstract}
Studies of cell development in artificial microenvironments can contribute to understanding a series of physiological mechanisms that might be influenced by geometrical features of the microenvironment itself. In this work we applied two-photon polymerization to fabricate threedimensional microenvironments composed of a matrix arrangement of microstructures (circular and square cross-sections pillars) with distinct spacing. Such microenvironments were used to evaluate the growth of Michigan Cancer Foundation-7 (MCF-7) cells that are commonly used as a model system to investigate fundamental aspects of the tumor biology. Our results reveal that the cell density decreases as the distance between structures in the environment is increased. Additionally, cell growth shows slightly better results for the microenvironments composed of circular crosssection structures.

DOI: 10.2961/jlmn.2014.03.0013
\end{abstract}

Keywords: Two-photon absortion; microfabrication; photopolimerization; Michigan Cancer Foundation-7; Green Fluorescent Protein

\section{Introduction}

Cell growth monitoring is fundamental for understanding a variety of physiological processes. In order to replicate natural cell environments, it is necessary to produce structures with accurately defined features, since those can influence the attachment, migration, and proliferation of cells [1-5]. One method that has been recently explored to fabricate microenvironments for cell culture is the use two-photon polymerization (2PP) [1-5]. This method takes advantage of the spatial selectivity provided by two-photon absorption to fabricate complex three-dimensional structures, without geometrical limitation and with sub-micrometric resolution [6-10]. 2PP has been shown as a suitable approach for the fabrication of structures for bio-related studies, such as, for example, scaffolds to study cell viability, growth and migration [1-5, 11-13].

In this work we used $2 \mathrm{PP}$ to fabricate microenvironments with distinct geometrical features, to evaluate its influence on the development of cells, since only a few works addressing such issue have been reported in the literature $[1,2,4]$. Here, Michigan Cancer Foundation-7 (MCF-7) cells stably expressing GFP (Green Fluorescent Protein) [14] were cultured into microenvironments composed of a matrix of structures with different shapes (square and circular cross-section) and distinct spacing. MCF-7 is an epithelial cancer cell line, derived from breast adenocarcinoma that has been extensively used as a model system to investigate fundamental aspects of the tumor biology [15-17], as well as to test new treatments [18-20]. Since the fabricated microenvironments provided favorable conditions for cell development, the influence of microenvironments' geometrical features on cells growth was analyzed by transmission and fluorescence microscopies. Our results indicate a dependence of the cell growth on the spacing between the structures on the microenvironment, but not on its shape.

\section{Experimental}

The resin used for the microfabrication is composed by two three-acrylic monomers; tris(2-hydroxyethyl) isocyanurate triacrylate (Sartomer SR 368 - $50 \mathrm{wt} . \%$ ), and ethoxylated (6) trimethylolpropane triacrylate (Sartomer SR 499 - 50 wt.\%). While the first one provides hardness to the structure, the later reduces the shrinkage tensions upon polymerization, preventing deformations on the final structure [21]. As the photoinitiator for the polymerization process we used ethyl-2,4,6- trimethylbenzoyl phenylphosphinate, commercially known as Lucirin TPO-L [21]. The monomers are mixed to the photoinitiator for $1 \mathrm{~h}$ to obtain a homogeneous solution.

Microstructures were produced by two-photon polymerization (2PP) using a Ti:sapphire laser oscillator at 
$82 \mathrm{MHz}$ and delivering $100 \mathrm{fs}$ pulses, centered at $790 \mathrm{~nm}$. The laser beam is focused into the resin using a microscope objective with $0.85-\mathrm{NA}(60 \times)$. The intensity of femtosecond pulses at the focal volume is high enough to induce two-photon absorption and locally initiate the radical polymerization. The laser is scanned in the $\mathrm{x}$ $y$ direction by a pair of movable mirrors, while the sample's axial (z) positioning is performed by a motorized stage. An illumination source and a CCD camera allow for real time monitoring of the polymerization. This experimental apparatus is described in details elsewhere [22, 23]. After polymerization, the sample is immersed in ethanol to wash away the uncured resin, leaving on the substrate only the fabricated microstructures.

Fabrication procedure consisted of producing a matrix arrangement of microstructures, with different shapes (cylinders and parallelepipeds) and spacing between them $(12,18,24$ and $30 \mu \mathrm{m})$. Such microstructures arrangements, here named microenvironments, were characterized using scanning electron microscopy (SEM; HITACHI microscope, model TM3000). The fabricated microenvironments were kept in ethanol for 1 day to leach out the unpolymerized toxic monomer, and subsequently rinsed with distilled water. Finally, the samples were sterilized by performing irradiation with UV-light for a period of 1 hour.

MCF-7 cells were transduced with lentiviral particles carrying the coding region of GFP. MCF-7 cells were cultured in minimum essential medium (MEM) containing $0.01 \mathrm{mg} / \mathrm{ml}$ of human recombinant insulin and fetal bovine serum, to a final concentration of $10 \%$. The cells were kept in an atmosphere containing $5 \%$ of $\mathrm{CO}_{2}$ and temperature of $37{ }^{\circ} \mathrm{C}[24,25]$. We evaluated cell development into the fabricated microenvironments by monitoring fluorescence and transmission optical microscopy images (ZEISS, model LSM 700) for a period of $48 \mathrm{~h}$.

\section{Results and discussion}

Figure 1 shows scanning electron microscopy (SEM) images of typical microenvironments composed of microstructures with square (a) and circular (b) crosssections that are separated, in this case, by $24 \mu \mathrm{m}$. The SEM macrographs in Fig. 1 reveal that the microenvironments exhibit good definition and physical integrity. For all microenvironments fabricated (with structures separation of $12,18,24$ and $30 \mu \mathrm{m})$, the square section has a lateral dimension of $20 \mu \mathrm{m}$, while the circular ones have a diameter of approximately $10 \mu \mathrm{m}$.
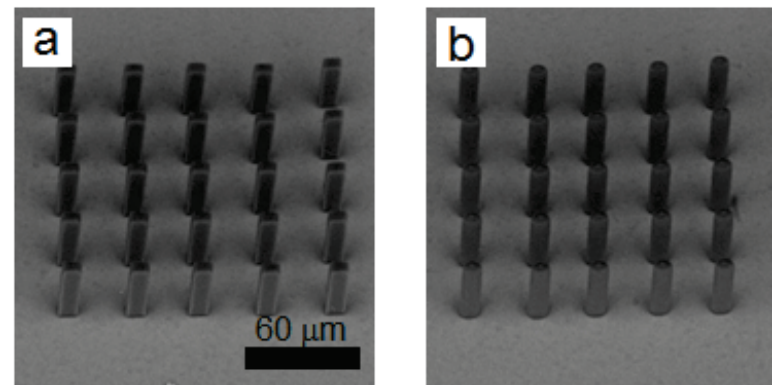

Fig. 1 SEM images of microenvironments with different geometries; square (a) circular (b) cross-sections.
The microenvironments were seeded at a cell density of $10^{4}$ cells $/ \mathrm{mL}$, after which we wait $24 \mathrm{~h}$ for cells attachment and proliferation, before starting taking images of the sample. The monitoring of the cell growth in the microenvironments was performed for two days (24 and 48 h) by optical microscopy. In Fig. 2 we show, as an illustration, a bright-field microscopy image of a microenvironment (square cross-section and spacing of 12 $\mu \mathrm{m})$ after $24 \mathrm{~h}$ of incubation. This result clearly shows the growth of MCF-7 cells nearby the structures, demonstrating the compatibility of the fabricated environments. Figure 2 also illustrates that, for some microenvironments, microstructures detach from the substrate during the cell culture.

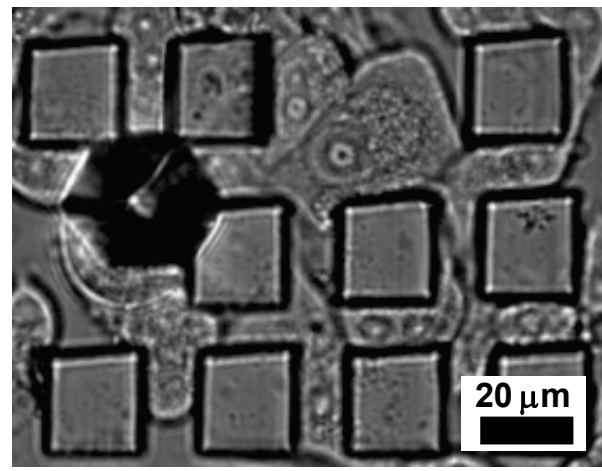

Fig. 2 Optical microscopy image of a microenvironment after $48 \mathrm{~h}$ of cell seeding.

The growth of MCF-7 cells in the distinct microenvironments was studied by analyzing optical microscopy images. Figure 3 shows a typical result of bright-field (a) and fluorescent (b) images of the cells (topview), obtained $48 \mathrm{~h}$ after seeding, for a microenvironment with $12 \mu \mathrm{m}$ of spacing. The microenvironments exhibit yellow fluorescence, resulting from the emission of the polymer, while cells present the characteristic green fluorescence due to the GFP expression. As it can be seen in Fig. 2, living cells are uniformly distributed throughout the microenvironment.

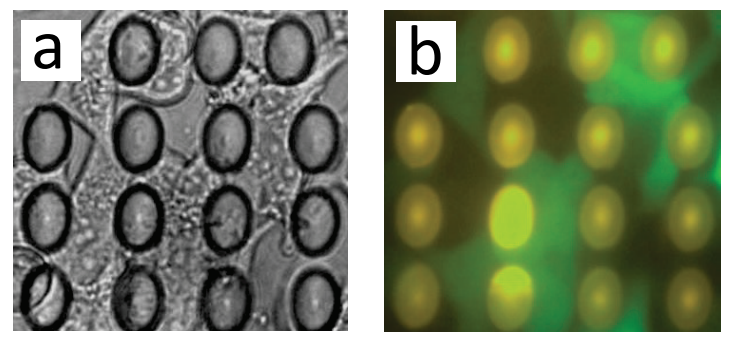

Fig. 3 Bright-field optical (a) and fluorescence microscopy (b) images of the $12 \mu \mathrm{m}$ spacing microenvironment with MCF-7 cells, after $48 \mathrm{~h}$ of incubation.

We studied the cell growth behavior in the distinct microenvironments by recording series of images similar to those presented in Fig. 3. From such images we determined the cell density (number of cells per area) for each one of the fabricated microenvironments. To obtain a better comparison between the results obtained for distinct 
microenvironments, that in fact present different shapes and spacing, our results are presented in terms of microenvironments' unit-cell area, defined as the free area between microstructures of a repetitive unit of the microenvironment, as illustrated in the inset of Fig. 4. The top axis of Fig. 4 indicates the corresponding microstructures spacing in the microenvironments.
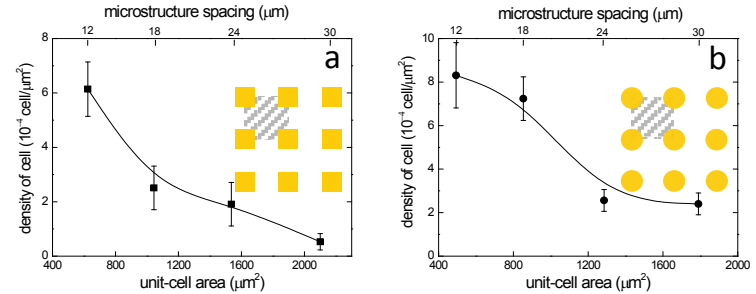

Fig. 4 (a) Cell density as a function of the unit cell area for the microenvironment composed of structures with square (a) and circular (b) cross-sections. The inset illustrates the unit cell are of the microenvironment. The lines along the points were drawn only to guide the eye.

Figure 4 show that, for both types of microenvironments (circular and square cross-section), the density of cells decreases as the unit-cell area of the microenvironment increases. Such trend is probably related to the fact that as the unit-cell area increases, the microstructures are farther apart, which diminishes cells contact with the structures in the microenvironment, hindering cells fixation and, consequently, growing. It is important to mention that the average area of the MCF-7 cell, determined in our experiments, is approximately 400 $\mu \mathrm{m}^{2}$. Although the results of Fig. 4 indicate that the cell density attained for both types of microenvironments are on the same order, distinct behaviors can be observed in Fig. 4 (a) and (b). While in Fig. 4(a), for the microenvironments with square cross-section, the cell density decreases almost linearly with the unit-cell area, for the ones with circular cross-section the decrease in cell density is less pronounced. It is interesting to note that for the circular cross-section environments (Fig. 4(b)), the density of cell seems to reach a plateau of $2.5 \mathrm{cell} / \mu \mathrm{m}^{2}$ for a unit-cell area of $1200 \mu^{2}$, for the range studied, dissimilar to the behavior observed in Fig. 4 (a) for the square cross-section microstructure. Therefore, such results indicate that the microenvironments composed of circular structures present slightly better features, which is probably related to a facilitated adhesion of cells to its surface.

\section{Conclusion}

We developed microenvironments, fabricated by $2 \mathrm{PP}$, composed of structures with circular and square shaped cross-section, and various spacing $(12,18,24$ and $30 \mu \mathrm{m})$. Such microenvironments showed to be biocompatible for cell growth. We have studied the growing of MCF-7 cells in distinct microenvironments, showing that the cell density decreases as the unit-cell area of the microenvironment increases, i.e., upon increasing the distance between structures in the environment, in the range tested here. Furthermore, circular cross-section microenvironments exhibits slightly better results for MCF-7 cells growth than the square ones, probably because it favors cell adhesion to its surface. Therefore, these results reveal, as a proof of principle, that the proper design of microenvironments, by tailoring their geometrical features, can be used to control the cell density in microenvironments, with direct implications in tissue engineering and microbiology studies.

\section{Acknowledgments}

The authors acknowledge financial support from FAPESP (Poc. 2011/12399-0, 2012/21807-7), CNPq and CAPES from Brazil

\section{References}

[1] Tayalia P, Mendonca CR, Baldacchini T, Mooney DJ, Mazur E. 3D Cell-Migration Studies using TwoPhoton Engineered Polymer Scaffolds. Advanced Materials. 2008 Dec 2;20(23):4494-8.

[2] Raimondi MT, Eaton SM, Lagana M, et al. Threedimensional structural niches engineered via twophoton laser polymerization promote stem cell homing. Acta Biomaterialia. 2013 Jan;9(1):4579-84.

[3] Claeyssens F, Hasan EA, Gaidukeviciute A, et al. Three-Dimensional Biodegradable Structures Fabricated by Two-Photon Polymerization. Langmuir. 2009 Mar 3;25(5):3219-23.

[4] Kapyla E, Aydogan DB, Virjula S, et al. Direct laser writing and geometrical analysis of scaffolds with designed pore architecture for three-dimensional cell culturing. Journal of Micromechanics and Microengineering. 2012 Nov;22(11).

[5] Turunen S, Kapyla E, Lahteenmaki M, Yla-Outinen L, Narkilahti S, Kellomaki M. Direct laser writing of microstructures for the growth guidance of human pluripotent stem cell derived neuronal cells. Optics and Lasers in Engineering. 2014 Apr;55:197-204.

[6] Gittard SD, Nguyen A, Obata K, Koroleva A, Narayan RJ, Chichkov BN. Fabrication of microscale medical devices by two-photon polymerization with multiple foci via a spatial light modulator. Biomedical Optics Express. 2011 Nov 1;2(11):3167-78.

[7] Cumpston BH, Ananthavel SP, Barlow S, et al. Twophoton polymerization initiators for three-dimensional optical data storage and microfabrication. Nature. 1999 Mar 4;398(6722):51-4.

[8] Kawata S, Sun HB, Tanaka T, Takada K. Finer features for functional microdevices - Micromachines can be created with higher resolution using two-photon absorption. Nature. 2001 Aug 16;412(6848):697-8.

[9] Lee K-S, Kim RH, Yang D-Y, Park SH. Advances in 3D nano/microfabrication using two-photon initiated polymerization. Progress in Polymer Science. 2008 Jun;33(6):631-81.

[10] Serbin J, Egbert A, Ostendorf A, et al. Femtosecond laser-induced two-photon polymerization of inorganicorganic hybrid materials for applications in photonics. Optics Letters. 2003 Mar 1;28(5):301-3.

[11] Ovsianikov A, Malinauskas M, Schlie S, et al. Threedimensional laser micro- and nano-structuring of 
acrylated poly(ethylene glycol) materials and evaluation of their cytoxicity for tissue engineering applications. Acta Biomaterialia. 2011 Mar;7(3):96774.

[12] Ovsianikov A, Gruene M, Pflaum M, et al. Laser printing of cells into 3D scaffolds. Biofabrication. 2010 Mar;2(1).

[13] Schlie S, Ngezahayo A, Ovsianikov A, et al. Threedimensional cell growth on structures fabricated from ORMOCER (R) by two-photon polymerization technique. Journal of Biomaterials Applications. 2007 Nov;22(3):275-87.

[14] Acedo P, Stockert JC, Canete M, Villanueva A. Two combined photosensitizers: a goal for more effective photodynamic therapy of cancer. Cell Death \& Disease. 2014 Mar;5.

[15] Bonofiglio D, Cione E, Qi H, et al. Combined Low Doses of PPAR gamma and RXR Ligands Trigger an Intrinsic Apoptotic Pathway in Human Breast Cancer Cells. American Journal of Pathology. 2009 Sep;175(3):1270-80.

[16] Crescenzi E, Varriale L, Iovino M, Chiaviello A, Veneziani BM, Palumbo G. Photodynamic therapy with indocyanine green complements and enhances low-dose cisplatin cytotoxicity in MCF-7 breast cancer cells. Molecular Cancer Therapeutics. 2004 May;3(5):537-44.

[17] Lois C, Hong EJ, Pease S, Brown EJ, Baltimore D. Germline transmission and tissue-specific expression of transgenes delivered by lentiviral vectors. Science. 2002 Feb 1;295(5556):868-72.

[18] Hartmann J, Getoff N. Effect of Free Radicals on the Biological Action of Genistein In Vitro and Synergism with Mitomycin C. Anticancer Research. 2009 Aug;29(8):3179-83.

[19] Komarova EA, Zelnick CR, Chin D, et al. Intracellular localization of p53 tumor suppressor protein in gamma-irradiated cells is cell cycle regulated and determined by the nucleus. Cancer Research. 1997 Dec 1;57(23):5217-20.

[20] Landers JE, Cassel SL, George DL. Translational enhancement of mdm 2 oncogene expression in human tumor cells containing a stabilized wild-type p53 protein. Cancer Research. 1997 Aug 15;57(16):3562-8.

[21] Baldacchini T, LaFratta CN, Farrer RA, et al. Acrylicbased resin with favorable properties for threedimensional two-photon polymerization. Journal of Applied Physics. 2004 Jun 1;95(11):6072-6.

[22] Correa DS, Cardoso MR, Tribuzi V, Misoguti L, Mendonca CR. Femtosecond Laser in Polymeric Materials: Microfabrication of Doped Structures and Micromachining. Ieee Journal of Selected Topics in Quantum Electronics. 2012 Jan-Feb;18(1):176-86.

[23] Otuka AJG, Almeida JMP, Tribuzi V, et al. Femtosecond Lasers for Processing Glassy and Polymeric Materials. Materials Research-IberoAmerican Journal of Materials. 2014 MarApr;17(2):352-8.

[24] ATCC. MCF - 7. [cited 2011; Available from: http://www.atcc.org/Products/All/HTB22.aspx\#85786B46AA23451B94BC5D45200673F7.
[25] Levenson AS, Jordan VC. MCF-7: The first hormoneresponsive breast cancer cell line. Cancer Research. 1997 Aug 1;57(15):3071-8.

(Received: August 1, 2014, Accepted: October 29, 2014) 